\title{
Correlated shadowing and fading characterization of MIMO off-body channels by means of multiple autonomous on-body nodes
}

\author{
Patrick Van Torre ${ }^{1,2}$, Peter Vanveerdeghem ${ }^{1,3}$, Hendrik Rogier ${ }^{1}$ \\ ${ }^{1}$ Department of Information Technology, Ghent University, Sint-Pietersnieuwstraat 41, B-9000 Ghent, Belgium \\ ${ }^{2}$ Department IT\&C, Ghent University, Valentin Vaerwyckweg 1, B-9000 Ghent, Belgium \\ ${ }^{3}$ Department ISP, Ghent University, Graaf Karel de Goedelaan 5, B-8500 Kortrijk, Belgium
}

\begin{abstract}
In off-body communication systems low-cost and compact transceivers are important for realistic applications. An autonomous off-body wireless node was designed and integrated onto a textile antenna. Channel measurements were performed for an indoor non line-off-sight $4 \times 2$ MIMO (Multiple-Input Multiple-Output) link using four off-body transmitting nodes and two similar fixed receiving nodes. The channel behavior is characterized as Rayleigh fading with lognormal shadowing and is fitted to a model determining fading and shadowing correlation matrices. The physics of the propagation is captured accurately by the model which is further used to simulate a link using diversity by means of Selection Combining, as implemented on the wireless nodes. The performance of measured and simulated links is compared in terms of outage probability level. The measurements and analysis confirm that the correlated shadowing and fading model is relevant for realistic off-body networks employing diversity by means of Selection Combining.
\end{abstract}

Index Terms-body-centric communication, textile antennas, diversity, MIMO, channel characterization.

\section{INTRODUCTION}

Off-body communication remains an important research domain and is a promising technology with applications in many fields, ranging from protective systems for rescue workers to fall-detection units for the elderly. The combination of this type of wireless communication with textile antennas allows unobtrusive integration into clothing. In particular at the higher frequency bands, such as the $2.45 \mathrm{GHz}$ ISM (Industrial Scientific and Medical) band, antennas are conveniently small compared to their usual deployment areas on the human body, thanks to the short wavelength, which is only $12 \mathrm{~cm}$.

As these communication systems are often used in indoor environments, the signals are subjected to multipath propagation resulting in severe fading, with additional shadowing by objects in the environment attenuating the signals. Specifically for off-body communication, significant additional shadowing of the signals by the human body itself is present (body shadowing). It is well-known that diversity techniques, employing multiple transmit and/or receive antennas, mitigate the effects of both fading and shadowing. The human body provides more than sufficient space to deploy multiple antennas for the $2.45 \mathrm{GHz}$ band. In particular, the use of front and back antennas will also counter the influence of body shadowing.
Measurements of off-body communication channels discussed in literature, including [1]-[6] and many others, often employ expensive and bulky equipment connected to antennas on the human body by means of coaxial cables. Further research into realistic practical systems unavoidably requires the development of more compact and fully autonomous systems. Such a wearable wireless network node was recently designed, developed and tested.

Using four off-body wireless nodes and two similar units in a fixed setup, a $4 \times 2$ MIMO channel measurement is performed in a NLoS (Non Line-of-Sight) propagation environment, which imposes the hardest conditions on the off-body link. The channel behavior in these conditions is assumed to be composed of Rayleigh fading with lognormal shadowing. The behavior of correlated shadowing for MIMO systems was also studied in [7]-[10].

Recently, a model that separates fading from shadowing based on the fitting of BER (Bit Error Rate) characteristics was published in [5]. In this model the envelope correlation matrix obtained by the measurement is processed in order to obtain separate fading correlation and shadowing correlation matrices. The behavior of the signals received by the wireless off-body nodes is captured accurately by this model, which is then used to simulate a MIMO link using diversity by means of SC (Selection Combining), a technique readily implemented on the wireless nodes.

In literature, wearable or implantable transceivers are described in [11]-[13]. A wireless inertial sensor node is presented in [14]. However, these systems are not intended to operate in an on-body multi-nodal network configuration and do not implement diversity. In [15] a 3-sensor node network monitors fatigue of soldiers, but the article focuses on a routing protocol. In [16] a network relying on SC-UWB as well as on IEEE802.15.4 is documented but it is intended for short range transmissions of only a few meter. To our knowledge no comparable publications are available documenting channel measurements and modeling with a realistic off-body MIMO system. The hardware infrastructure for the entire measurement setup is very compact, low-power and low-cost, making the results highly relevant to assess the performance of a realistic wireless off-body network in practical operation, which may be realized by exactly the same components. 


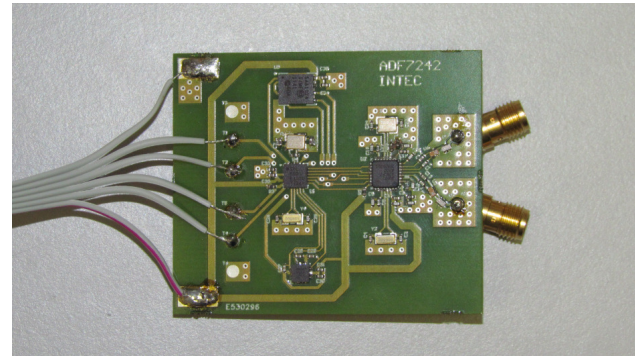

Fig. 1. A 2-input fully autonomous wireless node

\section{DESCRIPTION OF THE HARDWARE}

The autonomous wireless node, of which a prototype is displayed in Fig. 1, features dual receive inputs and a single transmit output. $4 \mathrm{MB}$ of nonvolatile memory is available to store acquired data. An advanced low-power microcontroller implements cooperation between multiple nodes, providing a compact, low-cost and low-power solution for the implementation of realistic off-body (sensor) networks. The node is directly connected to textile antennas integrated into the garment and can operate continuously for many hours relying on the power provided by a small battery. Full details of the wireless node are documented in [17].

Multiple off-body wireless nodes cooperate by means of a protocol where each node monitors the transmissions of the other nodes to synchronize the clock that determines its transmission slot. The nodes transmit repetitively in subsequent time slots controlled by their hard coded serial number. Packet numbers inserted into the data allow to extract timing information at the receiving end and to combine the right information in case of multiple receivers.

\section{MEASUREMENT SETUP}

\section{A. Selection combining MIMO system}

The wearable nodes will now be used for MIMO channel characterization. Earlier channel measurements performed by means of the Signalion testbed [5] used fully synchronous transmission on the multiple channels. However, in the lowcost system this is not possible and each node has to transmit in a different time slot.

In a system with single-chip SISO (single-input singleoutput) transceivers outputting only data and a received signal level for error-free packets, diversity can only be achieved by means of SC. High-performance techniques such as MRC (Maximum Ratio Combining) can not be employed as received signals before demodulation and detection are not available.

SC transmit diversity is achieved by transmitting the sensor data multiple times, in 4 subsequent time slots for each on-body node. The transmitted packets are received synchronously on the two receiving nodes, receiving each data block 8 times, over the different channels in the $4 \times 2 \mathrm{MIMO}$ setup. Finally for each group of received packets with equal data content, the packet with the highest received signal level is chosen by SC.

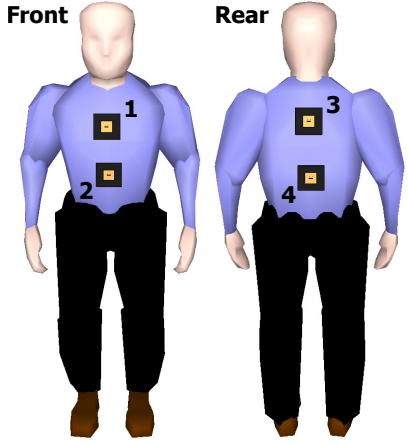

Fig. 2. Locations of the wireless nodes on the body

\section{B. Wearable four-node off-body transmit configuration}

A test person is equipped with a network of nodes implementing a fourth-order transmit diversity off-body channel using SC. Two transmitting nodes are worn on the front of the torso, stacked vertically at positions corresponding to the center of the chest and belly. At the back of the torso two transmitting nodes are worn in similar positions. The nodes are equipped with textile antennas as described in [18] and are placed in locations according to their ID number, as displayed in Fig. 2.

The four nodes transmit information sequentially at a rate of $33 \mathrm{~Hz}$, each node occupying a time slot related to its hardcoded ID-number. Synchronicity is automatically obtained and preserved throughout the transmission as the wireless nodes monitor each other's transmissions to adjust their internal clocks. Incremental packet numbers are inserted in the data for each transmitted frame. These numbers allow the receiver to keep track of packet loss and to combine the right packets to perform SC.

\section{Fixed two-node diversity receive configuration}

Diversity reception is realized by means of two identical nodes placed in a fixed setup, allowing to receive packets synchronously at both locations. Diversity reception is possible using a single node, but only one packet can be received at a given time, hence in such a setup each packet would have to be transmitted twice, as in [19]. The latter approach would lower the correlation between the receive antennas in the $4 \times 2 \mathrm{MIMO}$ link due to time diversity and therefore was not preferred here.

The textile antennas connected to both nodes are taped to cardboard boxes on top of desks in the indoor office area of which the floor plan is displayed in Fig.3. The antennas are at a height of $1.4 \mathrm{~m}$ above the floor, similar to the average height of the body-worn wireless nodes. The receive antennas are separated by a distance of $3 \mathrm{~m}$. They are marked RX1 and $\mathrm{RX} 2$ in the floor plan.

\section{Indoor propagation environment}

The test person performs a random walk in the indoor environment where the propagation between the TX and RX areas is NLoS (Non Line-of-Sight), as confirmed by previous measurement campaigns [19]. 


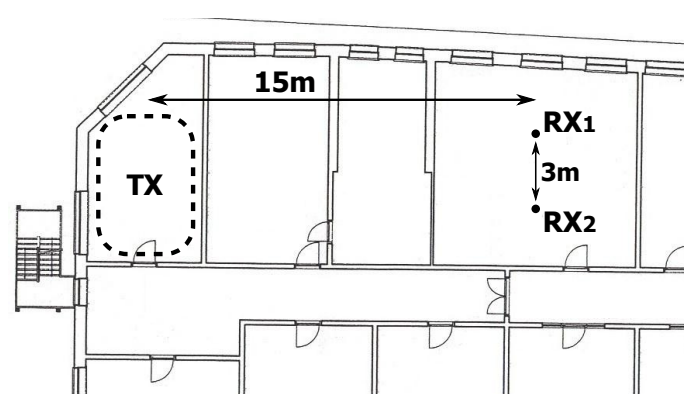

Fig. 3. Floorplan of the indoor measurement environment

The trajectory of the walking test person is confined within the TX area indicated in Fig.3, to provide a nearly constant path loss. The propagation is further influenced by small-scale fading and shadowing by objects such as office furniture.

\section{MeAsurement RESUlts}

\section{A. Recorded frames versus packet loss}

During an 8-minute off-body measurement a total of 4059 MIMO channel realizations were recorded, corresponding to 16236 packets transmitted by the walking person.

Some packet loss occurs using the integrated transceivers. Indeed, due to fading the received signal level is sporadically too low to be captured. The $4 \times 2$ MIMO link is composed of 8 SISO (single-input single-output) channels. The transmitreceive antenna combination and packet loss for these SISO channels is displayed in Table I.

\begin{tabular}{cccc} 
SISO\# & TX\#-RX\# & Packet loss [\%] & $\sigma_{s h}[\mathrm{~dB}]$ \\
\hline 1 & TX1-RX1 & 1.5 & 3.21 \\
2 & TX2-RX1 & 1.2 & 3.22 \\
3 & TX3-RX1 & 1.1 & 4.20 \\
4 & TX4-RX1 & 1.4 & 2.67 \\
5 & TX1-RX2 & 1.6 & 2.57 \\
6 & TX2-RX2 & 1.1 & 2.44 \\
7 & TX3-RX2 & 1.3 & 2.68 \\
8 & TX4-RX2 & 1.6 & 1.81
\end{tabular}

TABLE I

SISO CHANNEL PACKET LOSS AND SHADOWING STANDARD DEVIATION

\section{B. Shadowing correlation model}

To determine the correlation properties for the $4 \times 2 \mathrm{MIMO}$ link, only the frames where packets are received over all 8 SISO channels can be used. Therefore a reduced set of 3648 MIMO channel realizations, without any packet loss, is used in the process. However, results can also be compared to the performance of SC using all 4059 frames, as this technique is usable even when some SISO channels of the MIMO channel are missing. The difference in performance of $\mathrm{SC}$ with the full or reduced set of frames is insignificant, as shown further.

The shadowing variance $\sigma_{s h}^{2}$ is determined for the 8 SISO channels by means of the algorithm described in [5]. Table I lists the standard deviation $\sigma_{s h}$, expressed in $\mathrm{dB}$. The shadowing correlation matrix is determined according to the procedure also described in [5]. Its elements are adjusted by employing the alternation projections method [20] in order to obtain a valid (positive definite) correlation matrix, suitable for Cholesky decomposition [10], [21]. The estimated shadowing correlation coefficients are displayed in Table II for the SISO channel numbers as defined in Table I.

The estimated correlation coefficients for TX antennas on the same side of the body are always very high $(\geq 0.94)$, except for the front antennas when received by different receive antennas $(\approx 0.6)$. Correlations for front and back antennas are always very low $(\leq-0.88)$, demonstrating the complementarity of front and back antennas and their ability to mitigate body shadowing. The resulting match for the $4 \times 2$ BER characteristic is accurate, with a difference between measurement and model of only $0.35 \mathrm{~dB}$ at $\mathrm{BER}=10^{-3}$, as displayed in Fig. 4. The accuracy is comparable to the results obtained by applying the channel model to wireless off-body channel data generated by a measurement campaign performed with the more elaborate Signalion testbed in [5].

To illustrate the impact of the correlated shadowing model on the accuracy of the result, a curve is also plotted for a model accounting for correlated Rayleigh fading only, assuming that shadowing is not present. The envelope correlation matrix from the measurement is now directly used as fading correlation matrix. The resulting characteristic differs from the measurement by $0.9 \mathrm{~dB}$ at $\mathrm{BER}=10^{-3}$. This deviation is 2.6 times larger compared to the model with correlated shadowing included.

\begin{tabular}{|c|c|c|c|c|c|c|c|c|}
\hline $\mathrm{Nr}$. & 1 & 2 & 3 & 4 & 5 & 6 & 7 & 8 \\
\hline 1 & 1.00 & 0.95 & -0.88 & -0.88 & 0.55 & 0.63 & -0.88 & -0.88 \\
\hline 2 & 0.95 & 1.00 & -0.90 & -0.90 & 0.64 & 0.62 & -0.90 & -0.90 \\
\hline 3 & -0.88 & -0.90 & 1.00 & 1.00 & -0.88 & -0.90 & 1.00 & 1.00 \\
\hline 4 & -0.88 & -0.90 & 1.00 & 1.00 & -0.88 & -0.90 & 1.00 & 1.00 \\
\hline 5 & 0.55 & 0.64 & -0.88 & -0.88 & 1.00 & 0.94 & -0.88 & -0.88 \\
\hline 6 & 0.63 & 0.62 & -0.90 & -0.90 & 0.94 & 1.00 & -0.90 & -0.90 \\
\hline 7 & -0.88 & -0.90 & 1.00 & 1.00 & -0.88 & -0.90 & 1.00 & 1.00 \\
\hline 8 & -0.88 & -0.90 & 1.00 & 1.00 & -0.88 & -0.90 & 1.00 & 1.00 \\
\hline
\end{tabular}

SHADOWING CORRELATION MATRIX FOR THE 8 SISO CHANNELS

\section{Measurement and model for Selection Combining}

In the correlated shadowing model, shadowing correlation values are estimated based on calculated BER characteristics. However, the wireless nodes are unable to directly measure BER values. The nodes communicate using the IEEE 802.15.4 standard, including a CRC (Cyclic Redundancy Check). In case of an invalid checksum, the packet is considered not received and discarded. Hence, only error-free packets are processed by the integrated transceiver. No signal level is available for the rejected packets. However, for the result of SC diversity the unselected packets are not important. The recorded signal levels are perfectly usable to construct a CDF (Cumulative Distribution Function) and determine the outage probability for measurement and model. 


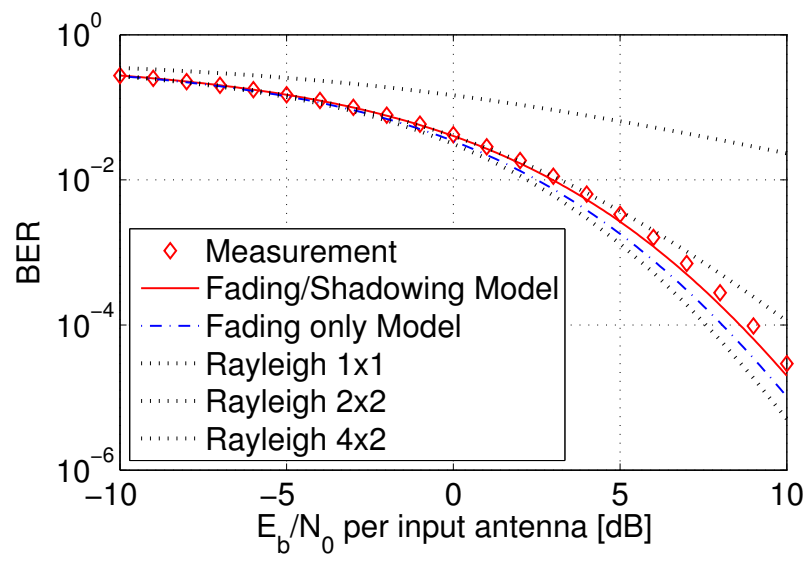

Fig. 4. $4 \times 2$ MIMO link; matching of BER characteristics for measurement and model; theoretical BER for uncorrelated Rayleigh fading channels

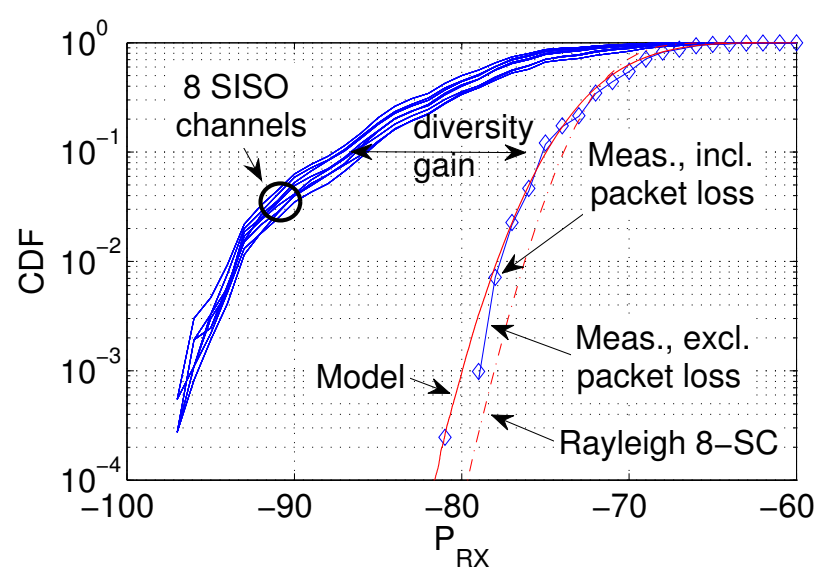

Fig. 5. $4 \times 2$ MIMO link: CDF for the measured SISO channels and for SC, for measurement and channel model; SC for unequal gain uncorrelated Rayleigh fading channels. Two overlapping curves are shown for the measurement, corresponding to the data set with (line) and without (markers) packet loss.

\section{Selection Combining in the presence of packet loss}

Interestingly, the difference between CDF curves for the reduced and full data sets is extremely small, as displayed in Fig. 5. Although 8-th order SC is implemented, sometimes one or more packets are lost, leaving less than 8-channels to combine. However, as explained in Section IV-C, effective 8-th order diversity gain is still obtained.

For probability levels lower than $10^{-3}$ the CDF for the measurement becomes very inaccurate due to the limited number of measured MIMO channel realizations. Taking into account the 4059 measured realizations, 4 or less of these realizations correspond to these low probability levels. Thanks to the $10^{7}$ simulated channel realizations, the CDF generated by the model represents the most accurate channel behavior in this area of the graph.

Table III displays the number of MIMO channel realizations in the measurement corresponding the a given number of SISO channels that are available for SC. The average signal level after SC, corresponding to the combination of this number of available SISO channels is listed as $\left\langle P_{R X, S C}\right\rangle$ (average linear power, converted to $\mathrm{dBm}$ after averaging). The value of $\left\langle P_{R X, S C}\right\rangle$ degrades by only $0.1 \mathrm{~dB}$ for 7 available channels, confirming the above reasoning. Note that the cases with combinations of 7 or 8 channels constitute $99.3 \%$ of the measured MIMO channel realizations. SC of less than 5 SISO channels never occurs in the measurement, illustrating the excellent reliability of the realistic $4 \times 2$ SC MIMO link.

\begin{tabular}{ccc} 
\# SISO channels & \# MIMO realizations & $\left\langle P_{R X, S C}\right\rangle[\mathrm{dBm}]$ \\
\hline 8 & 3648 & -69.00 \\
7 & 383 & -69.10 \\
6 & 25 & -69.55 \\
5 & 3 & -70.57 \\
$\leq 4$ & 0 & N/A
\end{tabular}

TABLE III

DISTRIBUTION OF THE NUMBER OF SIGNALS COMBINED BY SC on the average envelope for each measured SISO channel, which is equal to $\sigma \sqrt{\pi / 2}$. 


\section{CONCLUSION}

The autonomous on-body nodes provide an effective, lowcost and realistic solution to perform off-body MIMO channel measurements. As the nodes form a practical off-body MIMO platform, the results are highly relevant for a fully integrated system, with all the communication hardware integrated onto textile patch antennas, creating autonomous units which are then integrated into garment.

Despite the low-cost hardware, channel measurement data are captured reliably, at a high rate and with an accuracy of $1 \mathrm{~dB}$. The set of measured signal levels matches well to our earlier published shadowing and fading correlation model.

Because the on-body nodes employ single-chip transceivers, only Selection Combining diversity can be readily implemented. The outage probability for Selection Combining is simulated for the model and results are compared to the measurement, indicating that the correlated fading/shadowing model is suitable for simulating the performance of realistic Selection Combining off-body MIMO configurations. At the $10 \%$ outage probability level, the gain achieved by employing this MIMO technique is $11.0 \mathrm{~dB}$ when applying Selection Combining to the measured values, whereas $11.2 \mathrm{~dB}$ gain results from the model.

\section{ACKNOWLEDGMENT}

This research was funded by the Interuniversity Attraction Poles (IAP phase-VII) program, provided by the Belgian Science Policy Office (BELSPO).

\section{REFERENCES}

[1] S.L. Cotton and W.G. Scanlon, "Channel characterization for singleand multiple-antenna wearable systems used for indoor body-to-body communications", Antennas and Propagation, IEEE Transactions on, vol. 57, no. 4, pp. 980-990, April 2009.

[2] Y. Ouyang, D. J. Love, and W. J. Chappell, "Body-Worn Distributed MIMO System”, IEEE Trans. Vehicular Technology, vol. 58, no. 4, pp. 1752-1765, May 2009.

[3] I. Khan, P. S. Hall, A. A. Serra, A. R. Guraliuc, and P. Nepa, "Diversity Performance Analysis for On-Body Communication Channels at 2.45 GHz", IEEE Trans. Antennas Propag., vol. 57, no. 4, pp. 956-963, Apr. 2009.

[4] J. Karedal, A.J. Johansson, F. Tufvesson, and A.F. Molisch, "A measurement-based fading model for wireless personal area networks", Wireless Communications, IEEE Transactions on, vol. 7, no. 11, pp. 4575-4585, November 2008.

[5] Patrick Van Torre, Luigi Vallozzi, Lennert Jacobs, Hendrik Rogier, Marc Moeneclaey, and Jo Verhaevert, "Characterization of measured indoor off-body MIMO channels with correlated fading, correlated shadowing and constant path loss", Wireless Communications, IEEE Transactions on, vol. 11, no. 2, pp. 712-721, 2012.
[6] S. van Roy, F. Quitin, LingFeng Liu, C. Oestges, F. Horlin, J. Dricot, and P. De Doncker, "Dynamic channel modeling for multi-sensor body area networks", Antennas and Propagation, IEEE Transactions on, vol. 61, no. 4, pp. 2200-2208, 2013.

[7] Nam-Ryul Jeon, Kyung-Hoe Kim, Jung-Hwan Choi, and Seong-Cheol Kim, "A spatial correlation model for shadow fading in indoor multipath propagation", in Vehicular Technology Conference Fall (VTC 2010-Fall), 2010 IEEE 72nd, sept. 2010, pp. 1 -6.

[8] R.K. Sharma and J.W. Wallace, "Indoor shadowing correlation measurements for cognitive radio studies", in Antennas and Propagation Society International Symposium, 2009. APSURSI '09. IEEE, june 2009, pp. 1 -4 .

[9] S. S. Szyszkowicz, H. Yanikomeroglu, and J. S. Thompson, "On the feasibility of wireless shadowing correlation models", Vehicular Technology, IEEE Transactions on, vol. 59, no. 9, pp. $4222-4236$, nov. 2010.

[10] J.F. Monserrat, R. Fraile, and L. Rubio, "Application of alternating projection method to ensure feasibility of shadowing cross-correlation models", Electronics Letters, vol. 43, no. 13, pp. 724 -725, 212007.

[11] Yuan Gao, Yuanjin Zheng, Shengxi Diao, Wei-Da Toh, Chyuen-Wei Ang, Minkyu Je, and Chun-Huat Heng, "Low-power ultrawideband wireless telemetry transceiver for medical sensor applications", Biomedical Engineering, IEEE Transactions on, vol. 58, no. 3, pp. 768-772, 2011.

[12] Yanqing Zhang, Fan Zhang, Y. Shakhsheer, J.D. Silver, A. Klinefelter, M. Nagaraju, J. Boley, J. Pandey, A. Shrivastava, E.J. Carlson, A. Wood, B.H. Calhoun, and B.P. Otis, "A batteryless $19 \mu \mathrm{W}$ MICS/ISM-band energy harvesting body sensor node SoC for ExG applications", SolidState Circuits, IEEE Journal of, vol. 48, no. 1, pp. 199-213, 2013.

[13] Seulki Lee, Long Yan, Taehwan Roh, Sunjoo Hong, and Hoi-Jun Yoo, "A $75 \mu \mathrm{W}$ real-time scalable body area network controller and a $25 \mu \mathrm{W}$ ExG sensor IC for compact sleep monitoring applications", Solid-State Circuits, IEEE Journal of, vol. 47, no. 1, pp. 323-334, 2012.

[14] Yao-Chiang Kan and Chun-Kai Chen, "A wearable inertial sensor node for body motion analysis", Sensors Journal, IEEE, vol. 12, no. 3, pp. 651-657, 2012.

[15] N. Javaid, S. Faisal, Z.A. Khan, D. Nayab, and M. Zahid, "Measuring fatigue of soldiers in wireless body area sensor networks", in Broadband and Wireless Computing, Communication and Applications (BWCCA), 2013 Eighth International Conference on, 2013, pp. 227-231.

[16] Xiyu Lu, Xinlei Chen, Yong Li, Depeng Jin, Lieguang Zeng, and H.F. Rashvand, "ZebraBAN: a heterogeneous high-performance energy efficient wireless body sensor network", Wireless Sensor Systems, IET, vol. 3, no. 4, pp. 247-254, 2013.

[17] P. Vanveerdeghem, P. Van Torre, C. Stevens, J. Knockaert, and H. Rogier, "Dual-diversity wearable wireless node on a dual-polarized textile patch antenna", Submitted to IET SMT, Nov. 2013.

[18] L. Vallozzi, P. Van Torre, C. Hertleer, H. Rogier, M. Moeneclaey, and J. Verhaevert, "Wireless communication for firefighters using dualpolarized textile antennas integrated in their garment", Antennas and Propagation, IEEE Transactions on, vol. 58, no. 4, pp. $1357-1368$, April 2010.

[19] Patrick Van Torre, Peter Vanveerdeghem, Hendrik Rogier, and Ingrid Moerman, "Energy-efficient off-body communication nodes with receive diversity", IEEE SCVT, Namur, Belgium, Nov. 2013.

[20] N.J. Higham, "Computing the nearest correlation matrix, a problem from finance", IMA Journal of Numerical Analysis, vol. 22, no. 3, pp. 329, 2002.

[21] A.-P. Garcia-Ariza and L. Rubio, "Computing the closest positive definite correlation matrix for experimental MIMO channel analysis", Communications Letters, IEEE, vol. 15, no. 10, pp. 1038-1040, 2011. 